\title{
Study on Accessibility of Feeder Lines with Different Geometric Shapes
}

\author{
Qing Tang $\mathbb{D}^{1,2}$ and Peng Du $\mathbb{D}^{1,2}$ \\ ${ }^{1}$ School of Traffic and Transportation, Beijing Jiaotong University, Beijing 100044, China \\ ${ }^{2}$ Key Laboratory of Transport Industry of Big Data Application Technologies for Comprehensive Transport, Beijing 100044, China
}

Correspondence should be addressed to Peng Du; pdu@bjtu.edu.cn

Received 8 November 2019; Revised 12 May 2020; Accepted 15 May 2020; Published 1 June 2020

Academic Editor: Maria Vittoria Corazza

Copyright (c) 2020 Qing Tang and Peng Du. This is an open access article distributed under the Creative Commons Attribution License, which permits unrestricted use, distribution, and reproduction in any medium, provided the original work is properly cited.

\begin{abstract}
The performance of the urban rail transit system depends largely on the operation efficiency of its feeder system. An improved way is proposed in this paper to calculate the accessibility index which is used for evaluation of feeder lines, and both passengers' waiting time and the competition between different access modes are considered. Different from previous research, this paper focuses on whether and how the geometric shape differences affect the performance of fixed-route feeder lines. The impacts of line shape on accessibility are analysed by derived formulas for calculating accessibility under ideal conditions. The finding emerging from this study is that the accessibility of feeder lines differs obviously due to their shapes, given the same conditions of total line length, stop spacing, and vehicle fleet size. The service area of feeder lines with branches or a loop is closer to the rail station compared with that of linear lines, thus leading to fewer passengers served but shorter average travel time under specific routing plans. For further exploring the universality of the above finding, route alignment optimization models to maximize accessibility are built for different shaped lines. The optimal solutions of different shapes are obtained and compared in the cases of random generation to analyse the impacts of shapes on accessibility under different line length conditions.
\end{abstract}

\section{Introduction}

As the backbone of urban transportation, urban rail transit system undertakes the long-distance passenger transportation on the main corridors in a city. However, the service coverage of rail transit lines is limited due to relatively large stop spacing, so it is necessary to design efficient feeder systems to gather and distribute passengers. The feeder lines can be divided into fixed-route type and demand-response type according to whether their route and stops are fixed or not $[1,2]$. The former usually has specific geometric shape and routing plans and is more common in big cities with large population density, e.g., light rail lines used as feeder lines or conventional feeder bus lines. The latter has variable route alignment and stops adjusted by passengers' travel reservation and is more likely to be seen in less populated areas [3,4]. This paper focuses on fixed-route feeder lines and attempts to investigate whether the shape difference affects the performance of a feeder line and what factors contribute to these impacts.

Previous literature ignored the impacts of shape on the performance of feeder lines and paid particular attention to the optimization of route alignment and frequency to minimize both the cost of the operator and the travel time of passengers when designing a feeder line [5]. Chandra proposed to use accessibility index to measure the performance of a feeder line from the perspectives of passenger demand and passengers' average travel time [1], which can reflect more the nature of feeder line design, that is, how to collect more passengers to urban rail transit stations with higher efficiency. Based on Chandra's research, this paper redefines the accessibility index of fixed-route feeder lines, adding considerations of both passengers' waiting time and the relationship between passenger demand and access distance, to make it closer to reality. Different from previous studies, this paper focuses on the influence of different 
shapes or routing plans on the accessibility of fixed-route feeder lines. The analysis has been conducted from two perspectives. Firstly, referring to the study of Quadrifoglio and $\mathrm{Li}$ [6], the accessibility calculation formulas for different shaped feeder lines under the condition of equal stop spacing are derived and applied to analyse the difference in accessibility influenced by shapes. Then, route alignment optimization models with the maximized accessibility as the goal for three typical shaped lines are built. The optimal solutions in the randomly generated cases are obtained and compared to verify the findings of impacts by shape types. According to the difference in accessibility, transportation planners can choose the best line shape in specific situations when designing fixed-route feeder lines.

This paper is structured as follows: Section 1 is the introduction of this paper; Section 2 reviews the previous literature; Section 3 describes the new definition of accessibility index of feeder lines; Section 4 analyses the impacts of shape difference on the accessibility of feeder lines; Section 5 explains the practical significance of the findings in this paper; conclusions are drawn in Section 6.

\section{Literature Review}

Accessibility is a complex and diverse concept, which can be traced back to the gravity model proposed by Reilly in the description of the commercial distribution in 1931 [7]. Previous literature has provided a plethora of definitions and calculation methods on accessibility, and different scholars have their understanding and utilization to achieve their specific research objectives. For it often introduced to express the interaction between transportation and land use, accessibility has long been discussed and used by transportation planners and policymakers to improve the quality of life and equity [8].

Accessibility can be defined as people's ability or ease to reach something such as destinations, activities, and services [9]. Hence, it is usually linked to the service equality of a transit system and widely used in transportation research [10]. In transit-specific research, the meanings of accessibility are traditionally distinguished by terms of "to transit" and "by transit" [11]. To-transit accessibility is defined as people's ability to access to transit system from origin, reflecting the coverage level of specific transit systems. For example, from individual-based perspective, it is usually measured as the space or time barrier between origin/ destination and bus stop or metro station [12], or the number of bus stops people can reach within a threshold time or distance [13]. Lessa et al. expressed accessibility as the ratio of the number of bus stops in the region to the total population of the same region [14]. From infrastructure-based perspective, the area or population covered within specific walking distance or time cost is also a measurement of to-transit accessibility [15]. By-transit accessibility concerns the convenience of accomplishing specific activities by transit, so it focuses more on travel time, transfer times, and fare cost. Some scholars define it as the number of activities people can accomplish with space-time constraints [16]. Xu and Yang divided the city into several grids and defined bus accessibility as the number of grids that could be reached within two transfers in the bus network [17]. Curtis et al. used an accessibility index, which is defined as the number of population and jobs that a node could cover in an isochronous circle, to represent the level of transportation supply [18]. Also, Cui et al. defined accessibility as the ratio of the number of high-paying jobs to the total number of jobs within a certain commuting time range [19]. The gravity model is often used to measure by-transit accessibility, for it considered both attractiveness of destinations and passengers' travel cost [20-22]. Zhu and Liu and Merlin et al. defined accessibility based on the gravity model in their research to measure the impacts of new-built transportation infrastructure to the employed population $[23,24]$. Rather than only physical access to destinations or activities, a broad definition of transit accessibility also concerns about people's perception such as affordability, reliability, and safety. For example, built environment around bus stops and convenience of pedestrian affect people's willingness to take a bus $[25,26]$, so they can also be considered as accessibility indicators. As people's feelings about different parts of the journey time are not the same, the weighted average travel time is used as a measure of accessibility instead of actual travel time in some literature studies [20, 27, 28].

Although there is a wealth of research on transit accessibility, few studies apply this conception to the evaluation of the urban rail transit feeder system. The performance of a feeder line can be measured from two perspectives [10]. One is from a spatial perspective, using coverage indicators to reflect the number of people served by the feeder line, as the research did by Aklilu and Necha [15]. It is a kind of to-transit accessibility, for it concerns about the scope of feeder line services but ignored the time or other costs spent by passengers on the buses. The feeder system is expected to have high transport efficiency, and therefore, its operating speed in the main travel direction must be guaranteed, especially for the demand-response feeder lines [29]. The other is from the perspective of time, to calculate the time spent by passengers from feeder lines stop to the main rail transit station, reflecting the transport efficiency. Quadrifoglio and Li derived a formula for calculating the average travel time of the residents served by linear fixedroute feeder lines to reflect the service level of the feeder line [6]. According to Quadrifoglio and Li, Chandra et al. first proposed the definition of accessibility of feeder lines which is the ratio of the total service population $P$ to the weighted average travel time $E[T]$ based on the gravity model from the both two perspectives [1] and derive its calculation formula for linear fixed-route lines which has the following form:

$$
\begin{aligned}
\text { accessibility } & =\frac{P}{E[T]^{\beta}}, \\
P & =\rho W d(n-1), \\
E[T] & =E\left[T_{\mathrm{rd}}\right]+\gamma E\left[T_{\mathrm{wk}}\right],
\end{aligned}
$$




$$
\begin{gathered}
E\left[T_{\mathrm{rd}}\right]=\frac{N}{2}\left(\frac{d}{v}+t\right), \\
E\left[T_{\mathrm{wk}}\right]=\frac{1}{v_{\mathrm{wk}}}\left(\frac{W}{4}+\frac{d}{4}\right),
\end{gathered}
$$

where $\rho$ is the population density of the service area, $d$ is the average stop spacing, $n$ is the number of stops of feeder lines, $W$ is the width of the line service area, $E[T]$ is the average travel time of all passengers taking feeder buses from home to rail transit station, which is composed of the average riding time $E\left[T_{\mathrm{rd}}\right]$ and the average walking time $E\left[T_{\mathrm{wk}}\right], \gamma$ is the weight coefficient of walking time, $v_{\mathrm{wk}}$ is the average walking speed, and $\beta$ is the impedance decay factor.

The method proposed by Chandra et al. is suitable for the evaluation of feeder lines for it considers both the service scope and passengers' time cost [1]. It can be considered as a by-transit accessibility indicator for evaluating the performance of a feeder line or a to-transit accessibility indicator to evaluate the coverage level of the main rail transit system. However, it missed two factors that are necessary to evaluate the performance of feeder lines from the viewpoint of operation. First, the assumption of equal potential demand at all stops does not consider the competition between different feeder modes. For example, the bicycle mode will have a higher split rate in the areas near the rail transit station, where the demand for feeder bus lines is low [30, 31]. Moreover, the average waiting time is not included as part of the average travel time in their definition. The journey on feeder buses is usually short, so waiting time accounts for an unneglectable large proportion of the total travel time. Therefore, the accessibility of feeder lines defined in Section 3 of this paper takes both of these two factors into consideration in order to make the results more realistic.

This paper focuses on the impacts of different shapes or routing plans on the accessibility of fixed-route feeder lines. There are few studies on the accessibility of feeder lines in the previous literature, and the research on fixed-route feeder lines does not consider the influence of line shape. The reason why ignoring line shape may be that previous literature did not consider the competition between different feeder modes when evaluating feeder lines so that the impacts of shape difference is not obvious. This paper attempts to analyse these impacts realistically.

\section{Definition}

In this paper, a new by-transit accessibility indicator based on equation (1) is given to explore the impacts of shape on the performance of feeder lines. The calculation of $P$ and $E[T]$ is revised to make them closer to reality.

The population served is calculated as the product of line service area and population density in equation (2) so that the potential demand of all stops is equal and will not be affected by the distance between the stop and the main rail station when the stop spacing is constant. However, this hypothesis is not close enough to reality. Zhang et al. found that, with the increase of access distance, the split rate as well as passenger demand of feeder bus lines gradually increased as shown in Figure 1 [31]. When the distance between passengers and the main rail transit station is short, walking and cycling have a higher split rate [30]. This law is very important for the analysis of accessibility differences caused by shape differences. In this paper, $P$ is represented as the demand function $p(x)$ shown in equation (6), where $s$ is the maximum travel distance of cycling, $q$ is the maximum demand of a stop, and $x$ is the access distance:

$$
p(x)= \begin{cases}\frac{x}{s} \cdot q, & x<s, \\ q, & x \geq s .\end{cases}
$$

The average travel time is represented as the weighted sum of the average riding time and the average walking time in equation (4), but for short distance feeder lines, the proportion of waiting time is not small. Therefore, the average travel time of passengers defined in this paper is composed of three parts, average riding time, average waiting time, and average walking time which has the following form:

$$
E[T]=E\left[T_{\mathrm{rd}}\right]+\gamma_{\mathrm{wt}} E\left[T_{\mathrm{wt}}\right]+\gamma_{\mathrm{wk}} E\left[T_{\mathrm{wk}}\right],
$$

where $\gamma_{\mathrm{wt}}$ and $\gamma_{\mathrm{wk}}$ are the weight coefficients of waiting time and walking time, respectively. According to Wardman's research, their values could be $\gamma_{\mathrm{wt}}=1.77$ and $\gamma_{\mathrm{wk}}=1.69$ [32]. Though travel cost, comfort, safety (as discussed by Tabassum et al. [33]), and reliability of feeder lines can affect residents' travel choices $[34,35]$, residents in different areas accept them differently. Also, commuters are more sensitive to travel time which is the most important and universal measure of travel impedance. In this paper, the average travel time defined in equation (7) is taken as the only measurement of impedance. The impedance decay factor $\beta$ reflects people's willingness to travel by a specific transit system [20] and its value is usually greater than 1 [36]. Due to the lack of trip travel data, the value of $\beta$ is assumed in the analysis.

In summary, there are two differences between the accessibility index defined in this paper and that by Chandra et al. [1]. One is the way to calculate the number of people served. This paper assumes that the demand of each stop is related to the distance to the rail station which is represented as a passenger demand function $p(x)$. The other is the way to calculate average travel time, as waiting time is considered in this paper.

\section{Methodology and Results}

4.1. Feeder Lines with Different Shapes. Feeder lines have many different shapes in operation, and four of them are studied in this paper. Linear lines and loop lines are really common (see Figures 2(a) and 2(b)). Some light rail lines were also built as Y-shaped lines. Bukit Panjang LRT in Singapore has a special shape that can be regarded as a combination of a line and a ring. This kind of feeder lines is defined as spoon-shaped lines in this paper. The general view 


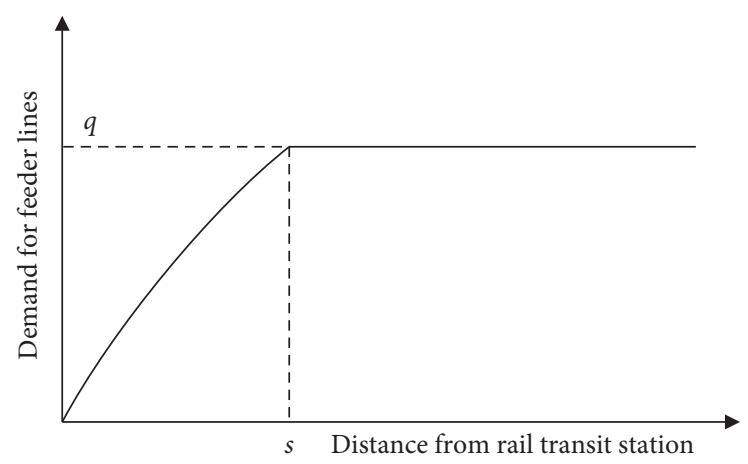

FIgURE 1: Demand varies with distance from stops to the main rail station.

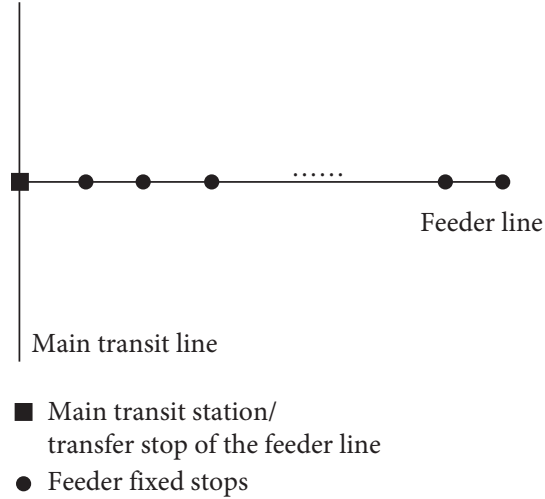

(a)

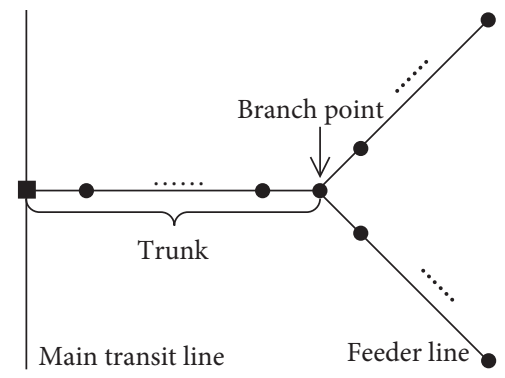

Main transit station/ transfer stop of the feeder line

- Feeder fixed stops

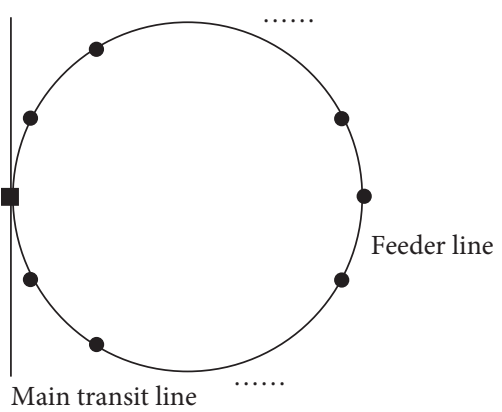

Main transit station/ transfer stop of the feeder line

- Feeder fixed stops

(b)

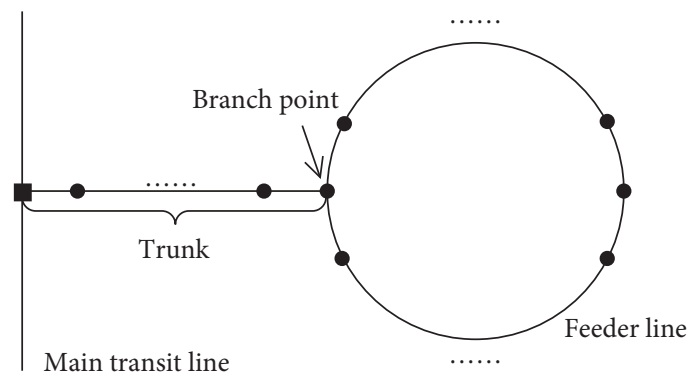

Main transit station/

transfer stop of the feeder line

- Feeder fixed stops

(d)

Figure 2: Shapes of feeder lines. (a) Linear line. (b) Loop line. (c) Y-shaped line. (d) Spoon-shaped line.

of Y-shaped lines and spoon-shaped lines is shown in Figures 2(c) and 2(d), respectively. The linear part near the main rail station is the trunk, while the branch part at the far end is spread out in two linear lines or one loop line. The intersection of the trunk part and the branch part is called the branch point.

The shape difference is mainly reflected in the difference in routing plans. Before deducing the accessibility calculation formulas of different shaped feeder lines, the routing plans of loop, Y-shaped, and spoon-shaped lines are defined as shown in Figure 3. It is supposed that all the three kinds of shaped lines have two routings, and the number of running vehicles on each routing is $N_{1}$ and $N_{2}$, respectively. In the derivation of the formulas, it is assumed that passengers travel following the two principles: (1) in the line with the shape of a loop, passengers choose the direction that has the less travel time to arrive at the main rail station; (2) if the travel time of two directions is equal, passengers can choose either direction. According to equation (2), the calculation formulas for accessibility of feeder lines with different shapes are shown in Table 1, where $p(x)$ is the passenger demand between the feeder bus stop and the main rail transit station and $x$ is the distance between them, which is calculated as feeder bus riding distance. The average walking time of 


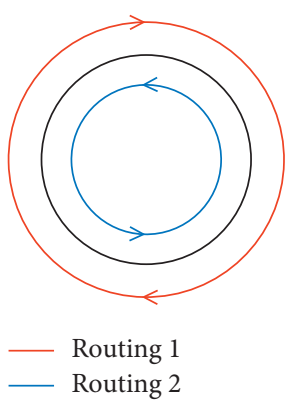

(a)

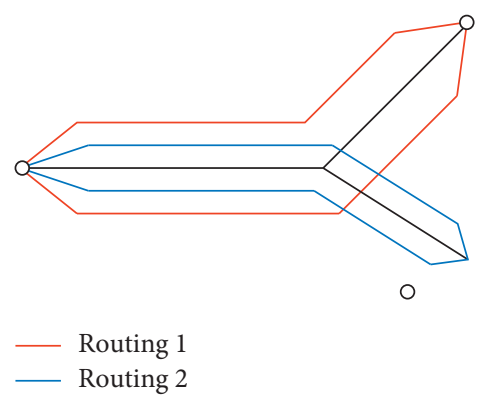

(b)

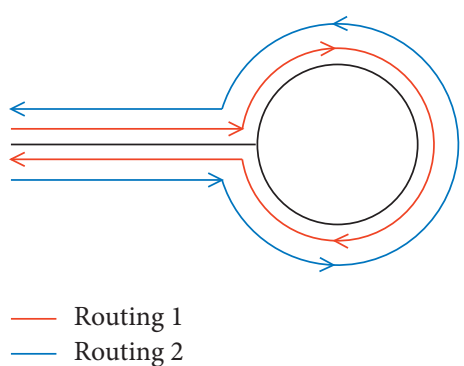

(c)

Figure 3: Routing plans of 3 different shaped lines.

passengers is calculated by equation (5). The other parameters have been defined in Section 3.

4.2. Comparative Analysis Based on Formulas. Previous literature has discussed the impacts of line length on the accessibility of feeder lines. The total length of the line is an important measure of the construction and operation costs. Therefore, this paper compares the accessibility of different shaped lines based on the same total length. Without loss of generality, it is assumed that the stop spacing is $500 \mathrm{~m}$, the number of vehicles in operation is $N=6$, the speed of vehicles is $v=40 \mathrm{~km} / \mathrm{h}$, the dwell time is $t=30 \mathrm{~s}$, the width of the service area is $W=600 \mathrm{~m}, s=3 \mathrm{~km}$, and $q=200$ in demand function $p(x)$. It is assumed that the length of the trunk of Y-shaped and spoon-shaped lines is $1 / 3$ of the total line length, and the number of vehicles in operation in different routings is equal. The comparison of accessibility of feeder lines with different shapes calculated by the formulas is shown in Figure 4.

As shown in Figure 4(a), with the increase of line length, the accessibility of four kinds of feeder lines increases due to the expansion of their service area. However, the slopes of their change curve vary differently. When the line is short, the accessibility of linear lines is relatively high, while that of loop lines is low. The accessibility of Y-shaped and spoonshaped lines is in between. When the line is long, the result is just the opposite. This is because the service area of the linear line is further away from the main rail transit station with the same line length, while that of the loop line is close. The linear line serves more distant residents, while the loop line serves the people who have shorter travel times on average. According to the definition of passenger demand function in equation (6), the demand of feeder lines at the near end is small, so the accessibility of loop lines is low when the line is short. With the increase of line length, the gap between the two shaped lines in the number of people served gradually approximates and the difference in average travel time becomes significant, so the accessibility of loop lines increases faster.

The Y shape and the spoon shape can be regarded as a combination of linear and loop shapes. Under the assumptions above, the accessibility of Y-shaped lines is a little higher than that of spoon-shaped lines, but the gap between them narrows with the increase of line length. It is similar to the comparison of linear lines and loop lines in the last paragraph. As shown in Figure 5, the average riding time and waiting time of spoon-shaped lines are both lower than those of Y-shaped lines with the same length, but the passenger volume (number of people served) of the latter is higher than that of the former. This is because spoon-shaped lines have fewer stops than Y-shaped lines under the same line length and stop spacing from a geometric point of view (Figure 6) and its service area is closer to the main rail transit station. Also, passengers served by the farthest stop of spoon-shaped lines can take buses in both directions, which decreases the average waiting time.

As shown in Figure 4(b), when $\beta=1.5$, there is a bigger difference in the accessibility of different shaped lines than when $\beta=1$. W When the lines are long enough, the accessibility of all the feeder lines except loop lines begins to decrease with the increase of line length, further indicating that the loop shape is more suitable for long feeder lines.

The ratio of the length of their trunk and branch also has impacts on the accessibility of Y-shaped lines and spoonshaped lines because it can affect passengers' average riding time and waiting time. For a spoon-shaped line, the longer the trunk is, the closer it is to a linear line. When the total length of the line is $6 \mathrm{~km}, 8 \mathrm{~km}, 10 \mathrm{~km}$, and $12 \mathrm{~km}$, respectively, the variation of accessibility with the proportion of the length of the trunk is shown in Figure $7(\beta=1.1)$, which is consistent with the rule revealed in Figure 4 . There is a rising trend when the ratio is greater than $80 \%$ in Figures $7(b-d)$ because at this time the decrement of the average waiting time is more than the increment of the average riding time with the unit increase of trunk length.

When the proportion of the trunk is 0 , the spoonshaped line is converted to a loop line, and the Y-shaped line is converted to a special linear line of which the transfer stop is in the middle. Obviously, this special linear line has high accessibility. The lines on both sides of the transfer stop are two branches. The variation of the accessibility of a linear line calculated by formulas in Table 2 with the location of the transfer stop (an intermediate stop) is shown in Figure 8(a). The accessibility of a linear line gets the highest value when the line length on both sides of the transfer stop is equal and is even higher than that of loop lines with the same line length (Figure 8(b)). 


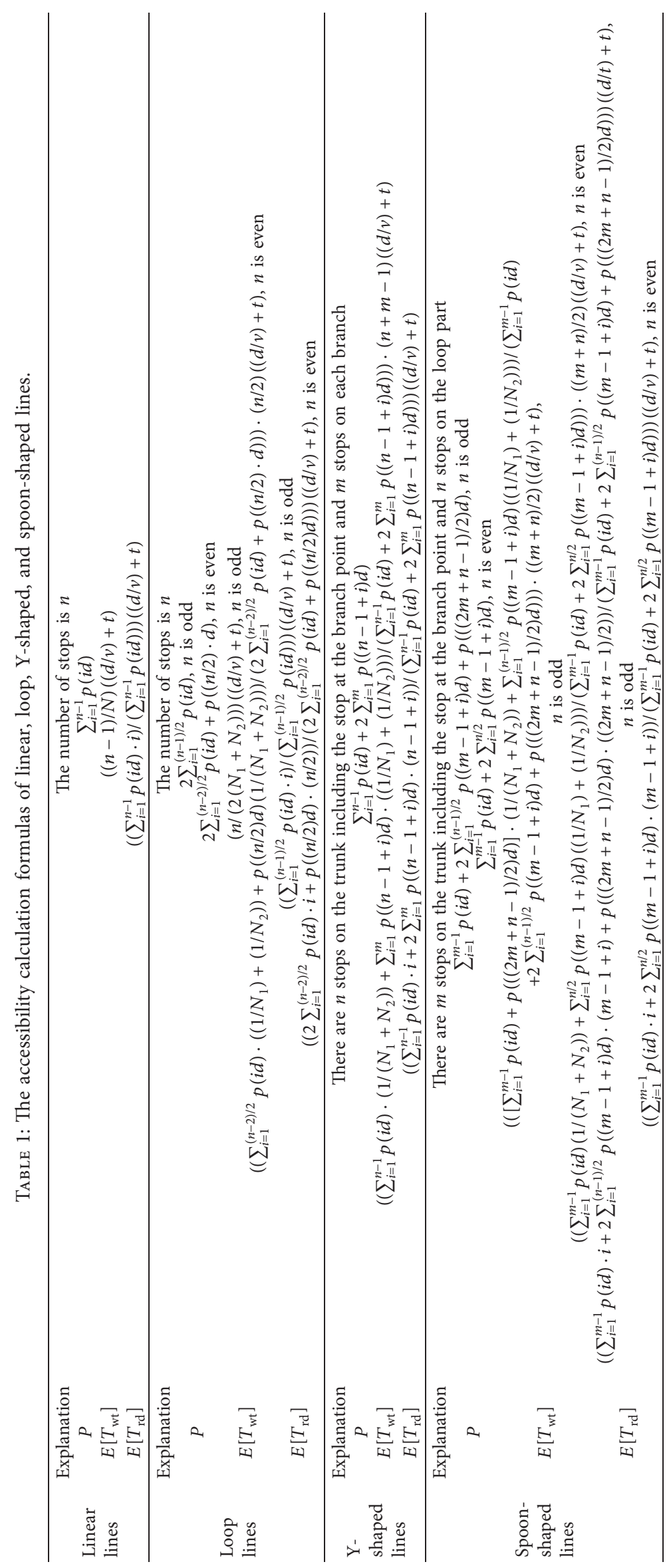




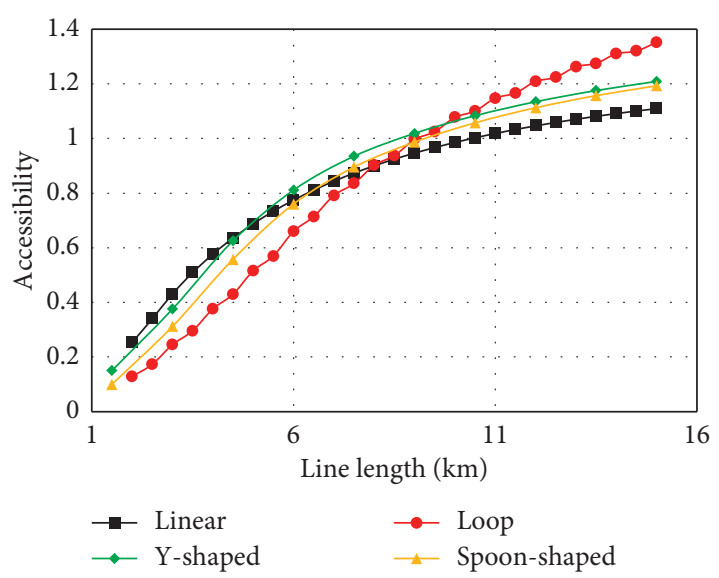

(a)

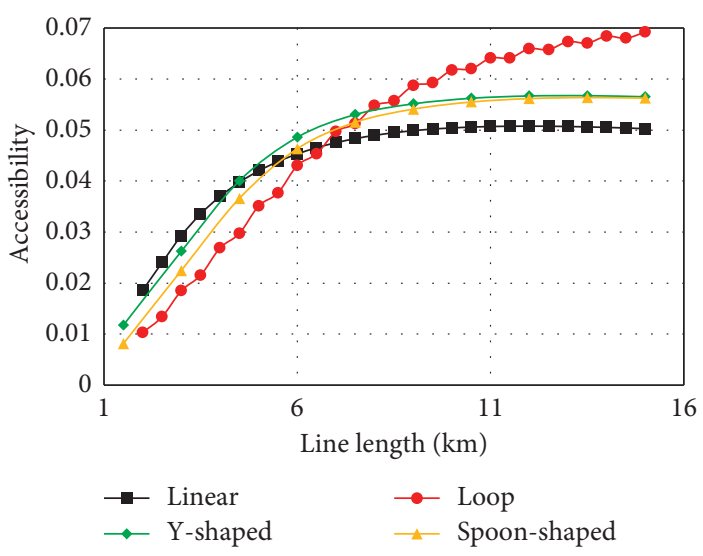

(b)

Figure 4: Comparison of accessibility of feeder lines with different shapes: (a) $\beta=1.1$ and (b) $\beta=1.5$.

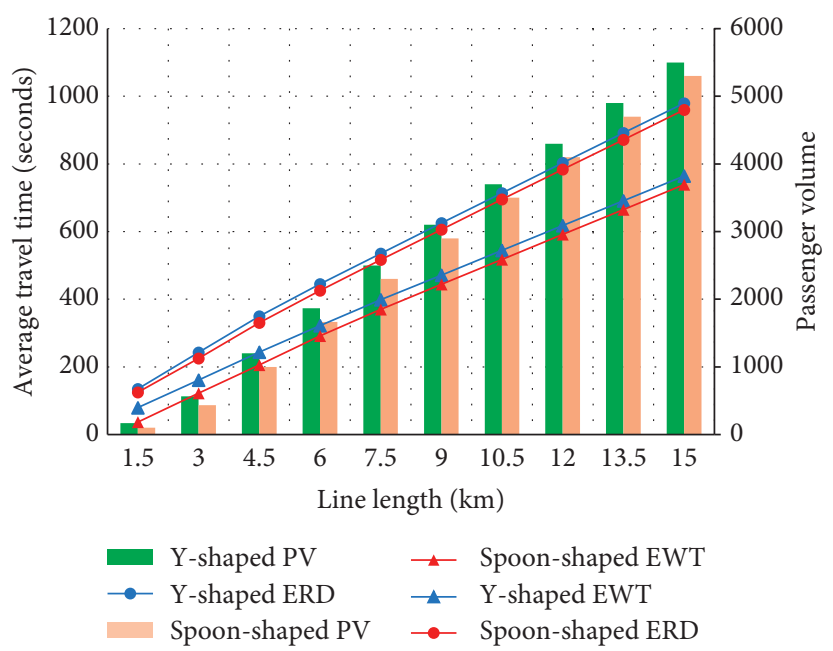

FIGURE 5: Comparison of Y-shaped lines and spoon-shaped lines (PV: passenger volume; EWT: average waiting time; ERD: average riding time).

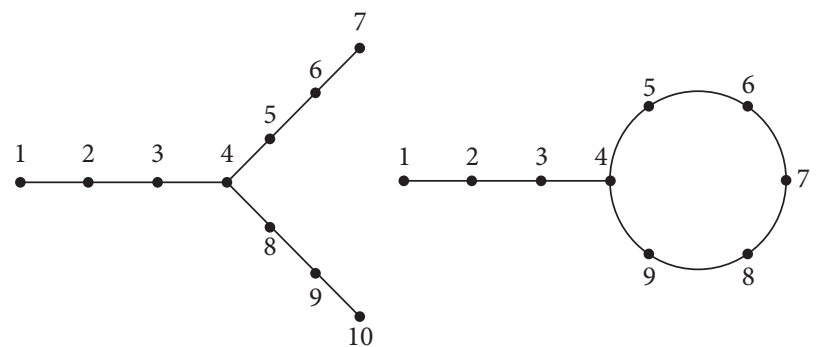

FIGURE 6: The two lines with the same line length but different number of stops.

4.3. Model. The discussion on accessibility in Section 4.2 is based on the condition that the stop spacing is constant and the demand of feeder lines strictly obeys the distribution of demand function $p(x)$, which is not close enough to reality. In this section, the impact of shape on accessibility is analysed from the perspective of optimization. Route alignment optimization models are built to maximize accessibility for different shaped lines and the optimal solutions of them are obtained and compared in the cases of random generation. The following analysis makes some concessions on the previous strict assumptions: (1) the passenger demand of each stop is no longer strictly related to the access distance; (2) the stop spacing is not constant; (3) and the feeder line no longer extends straight to the far end, and there are some detours at the near end, so the distance between the stop and urban rail station is not equal to the riding distance.

The models with the goal of maximized accessibility are built to determine the route alignment and stops position of feeder lines under the constraints of line length and the number of stops. The decision variable of the model is a list denoted as $r$, in which $r_{i}$ is the ith stop of the feeder line. $r_{1}=0$ represents that the first stop is designated as the transfer stop with the urban rail station. Other parameters involved in the model are listed in Table 3. It is assumed that the number of vehicles on each routing is the same, i.e., $N / 2$.

The objective function of the model is formed as follows: 


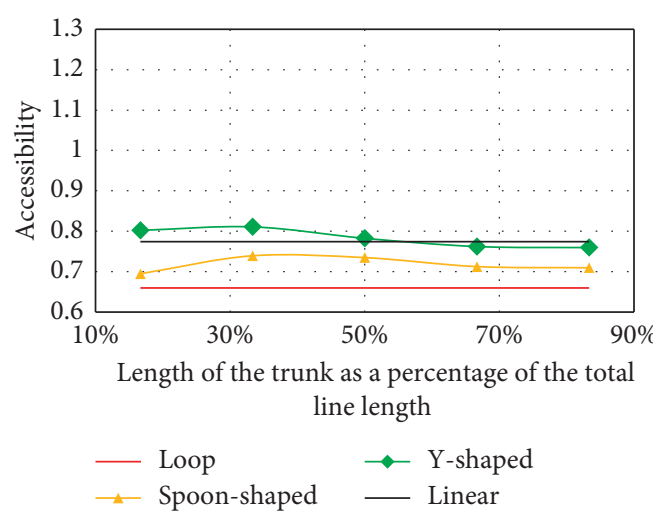

(a)

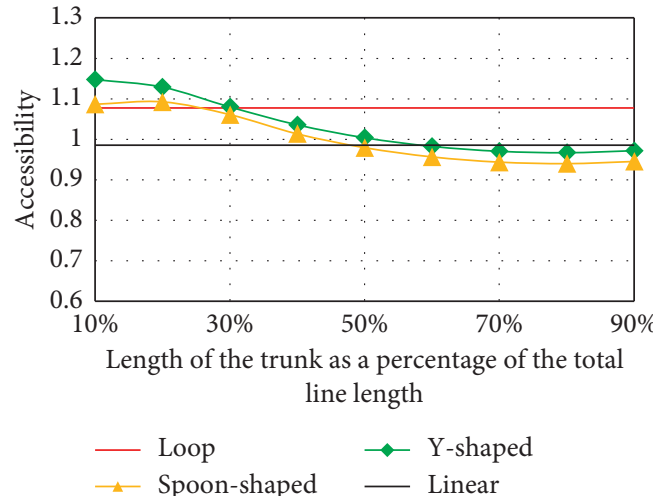

(c)

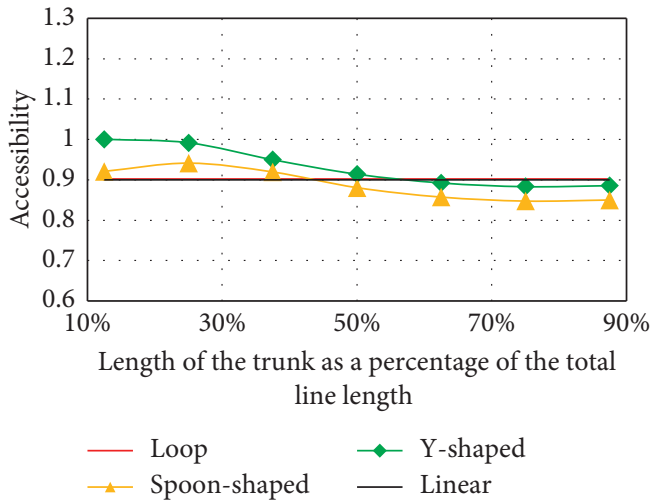

(b)

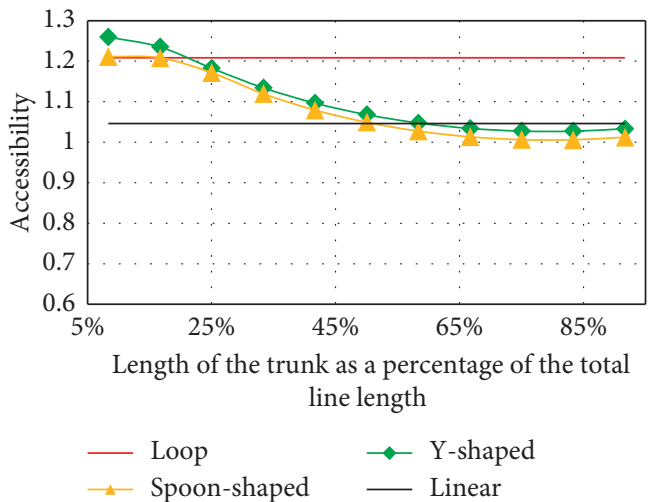

(d)

Figure 7: Variation of the accessibility with the length of the trunk as a percentage of the total line length. (a) Line length $=6 \mathrm{~km}$. (b) Line length $=8 \mathrm{~km}$. (c) Line length $=10 \mathrm{~km}$. (d) Line length $=12 \mathrm{~km}$.

TABle 2: The accessibility calculation formulas of the linear lines whose transfer stop is an intermediate stop.

\begin{tabular}{lc}
\hline Explanation & There are $n$ stops on one branch including the stop at the branch point and $m$ stops on the other one \\
\hline$P$ & $\sum_{i=1}^{n-1} p(i d)+\sum_{i=1}^{m} p(i d)$ \\
$E\left[T_{\mathrm{wt}}\right]$ & $((m+n-1) / N)((d / v)+t)$ \\
$E\left[T_{\mathrm{rd}}\right]$ & $\left(\sum_{i=1}^{n-1} p(i d) \cdot i((d / v)+t)+\sum_{i=1}^{m} p(i d) \cdot i((d / v)+t)\right) /\left(\sum_{i=1}^{n-1} p(i d)+\sum_{i=1}^{m} p(i d)\right)$ \\
\hline
\end{tabular}

$$
\max Z=\frac{\sum_{i=2}^{n} q_{i}}{\left(E\left[T_{\mathrm{rd}}\right]+\gamma_{\mathrm{wt}} E\left[T_{\mathrm{wt}}\right]+\gamma_{\mathrm{wk}} E\left[T_{\mathrm{wk}}\right]\right)^{\beta}} .
$$

However, the expressions of each part of the denominator of equation (8) are different for three shaped lines, which are listed as follows.

4.3.1. Linear Lines. The turnaround time of linear lines is given by

$$
\mathrm{T}_{\mathrm{Li}}=\frac{2}{v} \sum_{i=1}^{n-1} d_{r_{i}, r_{i+1}}+2(n-1) t
$$

The average waiting time of passengers served by all stops is as follows:

$$
E\left[T_{\mathrm{wt}}\right]=\frac{T_{\mathrm{Li}}}{2 N}=\frac{1}{v N} \sum_{i=1}^{n-1} d_{r_{i}, r_{i+1}}+\frac{n-1}{N} t .
$$

The average riding time is as follows:

$$
E\left[T_{\text {rd }}\right]=\frac{\sum_{i=2}^{n} q_{i}\left[(1 / v) \sum_{j=1}^{i-1} d_{r_{j}, r_{j+1}}+(i-1) t\right]}{\sum_{i=2}^{n} q_{i}} .
$$

According to the definition of equation (5), the average walking time is as follows. It is assumed that the service length of the last stop is twice the distance between it and the previous stop:

$$
E\left[T_{\mathrm{wk}}\right]=\frac{1}{v_{\mathrm{wk}}} \sum_{i=2}^{n-1}\left[\frac{1}{8}\left(d_{r_{i-1}, r_{i}}+d_{r_{i}, r_{i+1}}\right)+\frac{1}{4} W\right]+\frac{d_{r_{n-1}, r_{n}}+W}{4 v_{\mathrm{wk}}} .
$$

4.3.2. Loop Lines. There is a stop at the farthest end of the loop and its number is $n_{\mathrm{L}}$. The model assumes that passengers served by all stops can only travel in one direction.

The turnaround time of loop lines is given by 


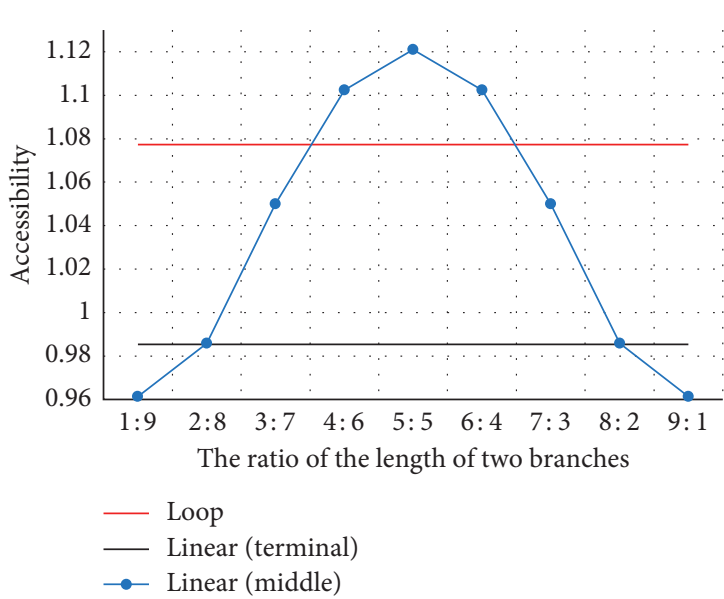

(a)

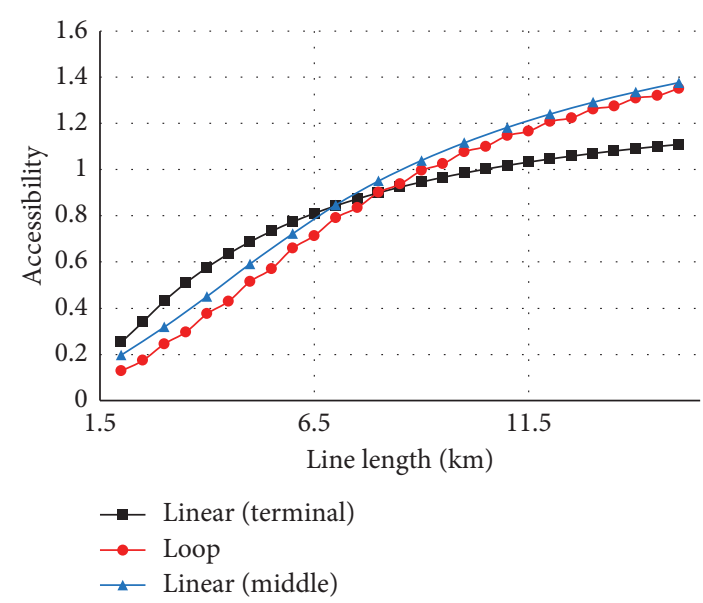

(b)

FIgURE 8: The impact of the location of the transfer stop on the accessibility of a linear line.

TABLE 3: The parameters involved in the model.

\begin{tabular}{l} 
Parameters \\
\hline$n$ \\
$N$ \\
$v$ \\
$\gamma_{\mathrm{wt}}$ \\
$\gamma_{\mathrm{wk}}$ \\
$v_{\mathrm{wk}}$ \\
$W$ \\
$\beta$ \\
$d_{i, j}$ \\
$q_{i}$ \\
$\qquad T_{\text {Lo }}=\frac{1}{v}\left(\sum_{i=1}^{n-1} d_{r_{i}, r_{i+1}}+d_{1, n}\right)+n t$.
\end{tabular}

The average waiting time is as follows:
The length of list $r$, i.e., the number of stops of the feeder line The total number of vehicles in operation

The average speed of the vehicles

The weight coefficients of waiting time The weight coefficients of walking time Walking speed

The width of the service area Impedance decay factor

The distance between stop $i$ and stop $j$

The passenger demand between stop $i$ and the rail station

$$
E\left[T_{\mathrm{rd}}\right]=\frac{\sum_{i=2}^{n_{\mathrm{L}}} q_{i} \cdot\left[(1 / v) \sum_{j=1}^{i-1} d_{r_{j}, r_{j+1}}+(i-1) t\right]+\sum_{i=n_{\mathrm{L}}+1}^{n-1} q_{i} \cdot\left[(1 / v) \sum_{j=n_{\mathrm{L}}}^{i-1} d_{r_{j}, r_{j+1}}+(n-i+1) t\right]+q_{n}\left((1 / v) d_{r_{1}, r_{n}}+t\right)}{\sum_{i=2}^{n} q_{i}}
$$

The average walking time is given by

$$
E\left[T_{\mathrm{wk}}\right]=\frac{1}{v_{\mathrm{wk}}} \sum_{i=2}^{n-1}\left[\frac{1}{8}\left(d_{r_{i-1}, r_{i}}+d_{r_{i}, r_{i+1}}\right)+\frac{1}{4} W\right]+\frac{d_{r_{1}, r_{n}}+W}{4 v_{\mathrm{wk}}}
$$

4.3.3. Spoon-Shaped Lines. The spoon-shaped line has two special stops. One is the stop at the branch point whose number is $n_{\mathrm{B}}$. The other is the stop at the farthest end of the loop part whose number is $n_{\mathrm{p}}$.
The turnaround time of spoon-shaped lines is given by

$$
T_{\mathrm{Sp}}=\frac{1}{v}\left(2 \sum_{i=1}^{n_{\mathrm{B}}-1} d_{r_{i}, r_{i+1}}+\sum_{i=n_{\mathrm{B}}}^{n-1} d_{r_{i}, r_{i+1}}+d_{r_{n}, r_{n_{\mathrm{B}}}}\right)+\left(n+n_{\mathrm{B}}-1\right) t
$$

The average waiting time is as follows:

$$
E\left[T_{\mathrm{wt}}\right]=\frac{\sum_{i=2}^{n_{\mathrm{B}}} q_{i}+2 \sum_{i=n_{\mathrm{B}}+1}^{n} q_{i}}{\sum_{i=2}^{n} q_{i}} \cdot \frac{T_{\mathrm{Sp}}}{2 N} .
$$

The average riding time is as follows: 
$E\left[T_{\mathrm{rd}}\right]=\frac{\sum_{i=2}^{n_{\mathrm{p}}} q_{i} \cdot\left[(1 / v) \sum_{j=1}^{i-1} d_{r_{j}, r_{j+1}}+(i-1) t\right]+\sum_{i=n_{\mathrm{p}}+1}^{n-1}\left[(1 / v)\left(\sum_{j=n_{\mathrm{p}}}^{i-1} d_{r_{j}, r_{j+1}}+\sum_{j=1}^{n_{\mathrm{B}}-1} d_{r_{j}, r_{j+1}}\right)+\left(n+n_{\mathrm{B}}-i\right) t\right]+q_{n}\left[(1 / v)\left(d_{r_{n}, r_{n_{\mathrm{B}}}}+\sum_{i=1}^{n_{\mathrm{B}}-1} d_{r_{i}, r_{j+1}}\right)+\left(n_{\mathrm{B}}-1\right) t\right]}{\sum_{i=2}^{n} q_{i}}$.

The average walking time is given by

$$
E\left[T_{\mathrm{wk}}\right]=\frac{1}{v_{\mathrm{wk}}} \sum_{i=2}^{n-1}\left[\frac{1}{8}\left(d_{r_{i-1}, r_{i}}+d_{r_{i}, r_{i+1}}\right)+\frac{1}{4} W\right]+\frac{d_{r_{n_{\mathrm{B}}}, r_{n}}+W}{4 v_{\mathrm{wk}}} \text {. }
$$

The constraints for models of three different shaped feeder lines are listed as follows.

Equations (21) (23) are line length constraints for linear lines, loop lines, and spoon-shaped lines, respectively. $L_{\min }$ and $L_{\max }$ are the lower and upper bound of the line length:

$$
\begin{aligned}
& L_{\min }<\sum_{i=1}^{n-1} d_{r_{i}, r_{i+1}}<L_{\max }, \\
& L_{\min }<\sum_{i=1}^{n-1} d_{r_{i}, r_{i+1}}+d_{r_{n}, r_{1}}<L_{\min }, \\
& L_{\min }<\sum_{i=1}^{n-1} d_{r_{i}, r_{i+1}}+d_{r_{n}, r_{n_{B}}}<L_{\max } .
\end{aligned}
$$

The following represents the constraint of the number of stops, and $n_{\min }$ and $n_{\max }$ are the lower and upper bound, respectively:

$$
n_{\min } \leq n \leq n_{\max }
$$

For loop lines, the stop at the farthest end $r_{n_{\mathrm{L}}}$ meets:

$$
\sum_{i=1}^{n_{\mathrm{L}}-1} d_{r_{i}, r_{i+1}} \geq \sum_{i=n_{\mathrm{L}}}^{n-1} d_{r_{i}, r_{i+1}}+d_{r_{1}, r_{n}}, \sum_{i=1}^{n_{\mathrm{L}}-2} d_{r_{i}, r_{i+1}}<\sum_{i=n_{\mathrm{L}}-1}^{n-1} d_{r_{i}, r_{i+1}}+d_{r_{1}, r_{n}} .
$$

For spoon-shaped lines, the stop at the farthest end $r_{n_{\mathrm{p}}}$ meets:

$$
\sum_{i=n_{\mathrm{B}}}^{n_{\mathrm{p}}-1} d_{r_{i}, r_{i+1}} \geq \sum_{i=n_{\mathrm{p}}}^{n-1} d_{r_{i}, r_{i+1}}+d_{r_{n}, r_{n B}}, \sum_{i=n_{\mathrm{B}}}^{n_{\mathrm{p}}-2} d_{r_{i}, r_{i+1}}<\sum_{i=n_{\mathrm{p}}-1}^{n-1} d_{r_{i}, r_{i+1}}+d_{r_{n}, r_{n B}} .
$$

4.4. Solving Algorithm. With the increase of stops, the number of alternative paths increases exponentially for $\mathrm{Ve}$ hicle Routing Problem (VRP) as an NP-hard problem. There are many methods to solve this kind of problem, which can be divided into precise algorithms and heuristic algorithms. The former is limited in practical application because of the large amount of computation. Considering the representation of the decision variables of the above model, simulated annealing algorithm is adopted in this paper.

Under the condition that every bus stop is numbered in the study region, the solution is expressed as a list when designing the algorithm, such as $[2,4,5,7,9,10,13,1,6,15$,
3], which records the number and sequence of the feeder stops except the transfer stop. The solutions of linear lines and loop lines are represented as one-dimensional lists, while those of spoon-shaped lines are represented as twodimensional lists, such as $[[1,4,5,7,9,10,13,8,6,15,3]$, [2]]. The number in the second sublist denotes how many stops in the first sublist are at the trunk. In this example, the number 2 means that $[1,4]$ is the stop sequence of the trunk and $[5,7,9,10,13,8,6,15,3]$ is that of the loop part.

The steps of simulated annealing algorithm are designed as follows:

Step 1: an initial solution list $r^{0}$ is generated. Set the current solution $r^{i}=r^{0}$, iteration step number $k=0$, and the current temperature $t_{\mathrm{k}}=t_{\max }$.

Step 2: the neighbourhood search is conducted to find some neighbourhoods $r_{j}$. The search process consists of 4 steps:

Step 2.1: two stops in the current solution are selected randomly and exchanged with two stops outside the solution.

Step 2.2: a stop is randomly added to or removed from the current solution.

Step 2.3: for spoon-shaped lines, a third step is required. The number in the second sublist randomly adds or subtracts 1 .

The Steps 2.1 2.3 are all conducted with certain probabilities.

Step 2.4: the order of stops in the current solution is rearranged to optimize the route alignment. In order to improve the calculation efficiency, Nearest Neighbour method is adopted which is shown in Figure 9. In an unordered collection of stops, firstly the stop closest to the rail station is chosen as the second stop of the feeder line (represented by the first number in list $r$ ). Then, the other stop closest to the second stop is chosen as the third stop of the feeder line, and so on.

For each neighbourhood $r_{j}$, the difference in fitness $\Delta f_{i j}$ is equal to $f\left(x_{j}\right)-f\left(x_{i}\right)$. If $\Delta f_{i j} \geq 0$ or $\exp \left(\Delta f_{i j} / t_{\mathrm{k}}\right)>\operatorname{random}(0,1)$ (a random decimal between 0 and 1), $r^{i}=r^{j}$.

Step 3: $k=k+1, t_{\mathrm{k}}=a \cdot t_{\mathrm{k}}$ ( $a$ is the cooling coefficient and $0<a<1$ ).

Step 4: repeating Steps 2 and 3 until the terminal condition is met.

4.5. Case Analysis. As is shown in Figure 10, a service area of a planned feeder system to a metro station is taken as an example for case analysis. It is a $4 \mathrm{~km} \times 4 \mathrm{~km}$ rectangular area with the station, the red point, at the bottom left origin, and 


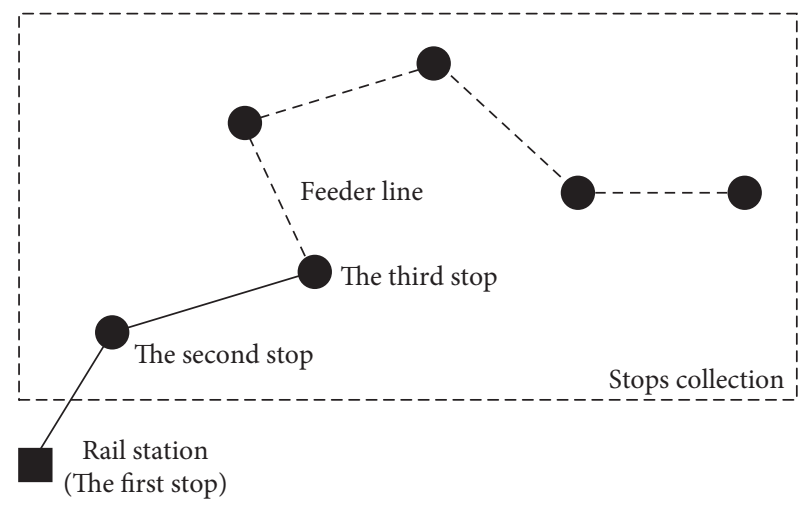

FIGURE 9: Sorting of the stops.

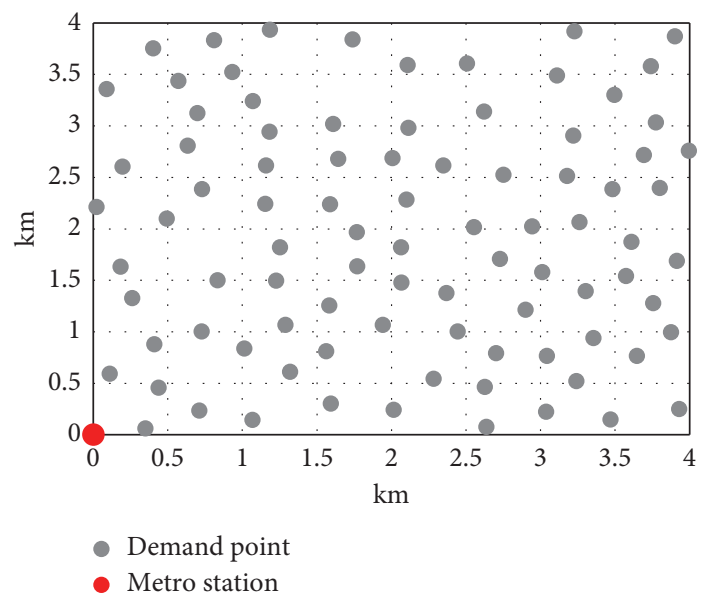

FIgURe 10: An example of the distribution of demand points.

89 (the density of the demand points is about the same as the density of bus stops within the third ring road of Beijing (872 stops in an area of $158.7 \mathrm{~km}^{2}$ ), which can be regarded as a representative of high population density cities in east Asia) passenger demand points, grey points, randomly generated and scattered in the area. The potential demand of each point obeys uniform distribution $U((1-\alpha) \cdot p(x),(1+\alpha) \cdot p(x))$, where $p(x)$ is the demand function defined by equation (6) and $x$ is the distance between the demand point and the metro station. It is assumed that $q=200, s=3 \mathrm{~km}$, and $\alpha=0.1$. Now a feeder bus line to serve some of those demand points needs to be designed extending from the metro station to the far end to express the impacts of the relationship between passenger demand and the access distance.

To ensure the universality of the results, 100 regions alike in Figure 10 are generated randomly, and simulated annealing algorithm is applied to solve the optimal solution of these regions with the following three conditions with different line lengths and the number of stops:

Condition 1: $L_{\min }=5 \mathrm{~km}, L_{\max }=6 \mathrm{~km}, n_{\min }=7$, and $n_{\max }=9$

Condition 2: $L_{\min }=8 \mathrm{~km}, L_{\max }=9 \mathrm{~km}, n_{\min }=10$, and $n_{\max }=12$
Condition 3: $L_{\min }=12 \mathrm{~km}, L_{\max }=13 \mathrm{~km}, n_{\min }=14$, and $n_{\max }=16$

The purpose of limiting the number of stops is to control stop spacings within a certain range. The parameters in the algorithm are set as follows: $t_{\max }=200$ and $a=0.98$. The iteration time is 100, and in each iteration, 200 neighbourhoods are searched. The values of the parameters in the model are the same as those in Section 4.2. The scatter plot of all optimal solutions of three shaped lines under three conditions is shown in Figure 11. Under condition 1 $(5 \sim 6 \mathrm{~km})$, the solutions of linear lines are better than those of the other two shaped lines on the whole. However, the solutions of spoon-shaped and loop lines are obviously better than that of linear lines under condition $3(12 \sim 13 \mathrm{~km})$.

By comparing the optimal solutions of the three different shaped feeder lines in each of the 100 service regions, the best solution is taken as the optimal solution of this region, and the shape distribution of the optimal solution of the 100 regions is shown in Figure 12. Under condition 1, 80 optimal solutions are obtained for linear lines, far more than the other two shaped lines. The spoon-shaped lines get the most optimal solutions under condition 2. Under condition 3, although the number of optimal solutions obtained by spoon-shaped lines is still larger than that of loop lines, the gap between the two is greatly narrowed, which indicates that the advantage of the loop lines in passengers' average travel time is reflected when the line is longer. The number of optimal solutions obtained by different shapes can be regarded as a measurement of their performance under specific line length constraint. This result verifies the findings of the accessibility comparison among linear, spoonshaped, and loop lines with different line lengths in the early part of this paper.

The experimental results with different values of the coefficient $\alpha$ under conditions 2 and 3 are shown in Figure 13. The number of optimal solutions obtained by loop lines in condition 3 is more than that in condition 2 with different values of $\alpha$, so the law revealed in Figure 12 is independent of $\alpha$. When $\alpha$ is large, the quantitative relationship between passenger demand and distance between the stop and the metro station is no longer strict, which is more consistent with reality. The experimental results show that the above findings are of universal applicability.

\section{Practical Significance}

The research on the effects of shape on accessibility can provide some inspiration for the planning and operation of feeder lines. Different shaped feeder lines have their own characteristics. Under the same line length, linear lines can extend further to serve the distant passengers, while loop lines mainly serve the passengers near the main rail transit station. The service areas of Y-shaped lines and spoonshaped lines are between the above two. Transportation planners can decide the transit routes and choose shapes according to specific residential distribution. However, as line length is not only a measure of operation and construction costs but also a reflection of service scope, feeder 


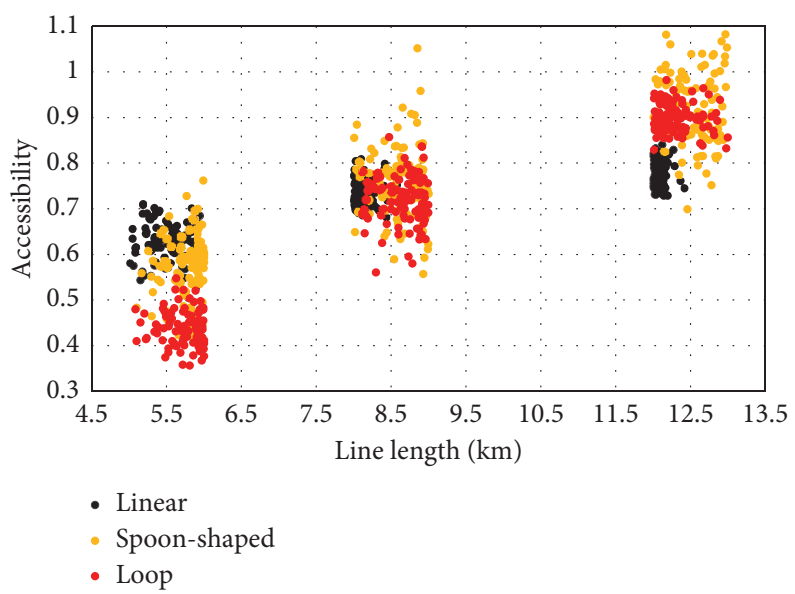

Figure 11: The scatter plot of all optimal solutions under three conditions.

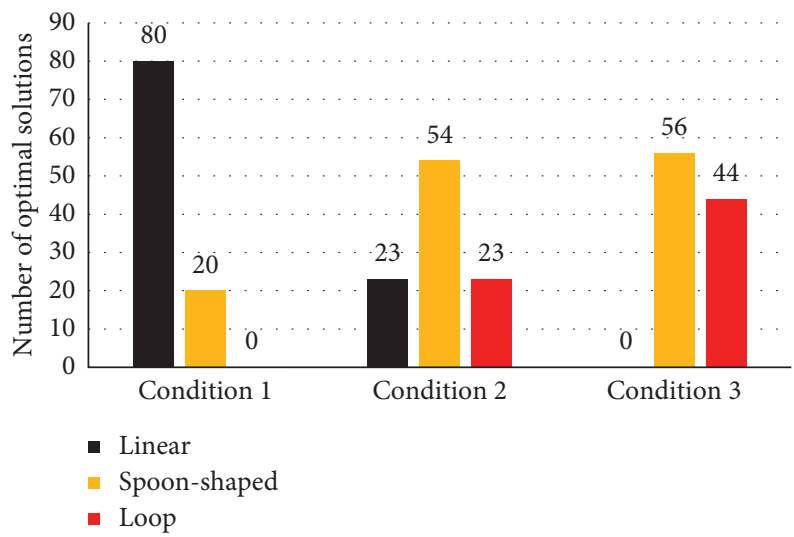

FIGURE 12: The shape distribution of the optimal solutions under three conditions.

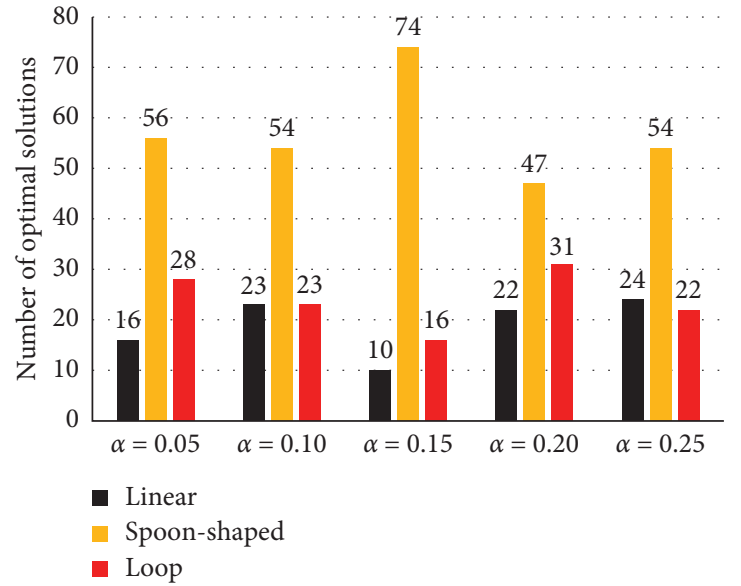

(a)

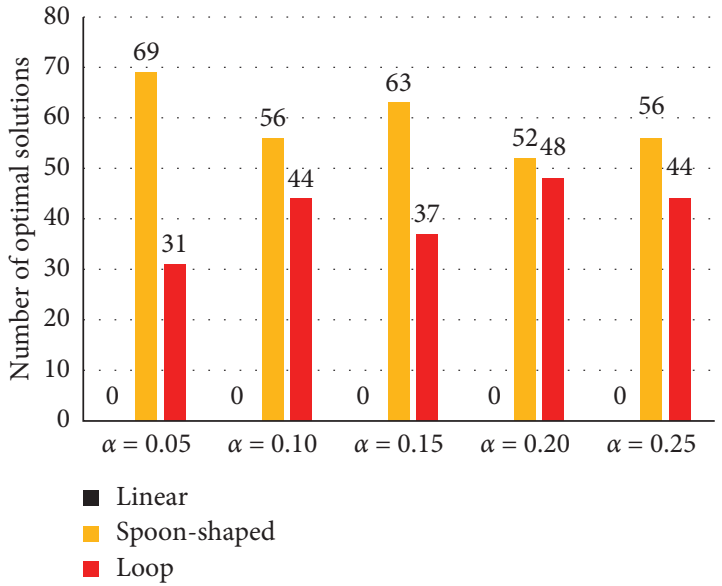

(b)

Figure 13: The results with different values of $\alpha$. (a) Condition 2. (b) Condition 3.

lines with different shapes have their suitable application scenarios in a general way. The service scope shared by each main rail station is small in the areas with high station density, so the feeder bus lines do not need to be built long.
At this time, linear feeder lines can achieve the highest accessibility as well as the travel efficiency of all passengers. In suburban areas, the density of main rail transit stations is small, so a feeder line needs to undertake the task of serving 
more passengers in a larger area. In this scenario, loop lines, spoon-shaped lines, Y-shaped lines, or other nonlinear lines can achieve higher accessibility. When designing feeder lines for main rail transit stations, it may be better to consider different shapes of lines in specific scenarios than only choosing linear lines, and the accessibility index proposed in this paper can be a good way for evaluation.

\section{Conclusions}

With the development of the automobile industry, urban transportation faces many problems such as traffic congestion and environmental pollution. Public transit managers are attempting to improve strategies to optimize the construction and operation of public transportation to reduce private car usage. Improving the first/last mile transport connectivity between urban rail stations and residences or offices is an important way to improve the competitiveness of public transportation.

The accessibility index based on the gravity model to evaluate feeder lines has been proposed by the previous literature. However, that definition of accessibility ignores passengers waiting time and the competition between different access modes, leading to its limitation in practical application. By considering these two important factors, this paper redefines an accessibility index of feeder lines based on the number of passengers and their average travel time. Moreover, the impacts of shape difference on the accessibility of feeder lines are analysed, which is not studied by previous articles. The accessibility calculation formulas of four typical shaped feeder lines are derived under the same conditions of line length, stop spacings, and vehicle fleet size, and comparative analysis of shape and line length is conducted with these formulas. Also, the optimization models of route alignment with maximum accessibility as the target for the three different shaped lines are built and solved, respectively, and the findings of the above comparative analysis are verified by comparing the solutions of different shaped feeder lines in the cases of random generation.

The main goal of feeder lines design is to maximize the connectivity performance at a certain cost. This paper assumes that the construction and operating costs of feeder lines are related to the length of lines and the number of stops. The conclusions are obtained by calculating and comparing the accessibility of feeder lines with different shapes and line lengths. There are differences in the accessibility of four typical shaped feeder lines in the experiment of this paper. Under the same condition of the line length, stop spacing, vehicle fleet size, and other given parameters, when the line is short, the accessibility of linear lines is the highest when comparing with other shaped lines and that of loop lines is the lowest. The accessibility of Y-shaped lines and spoon-shaped lines is somewhere in between. As the line length increases, the accessibility of spoon-shaped lines gradually reaches the highest. When the line length is long, the accessibility of loop lines gets the highest and that of linear lines becomes the lowest. The main reason for the above difference is that the service scope of the lines with the shape of branches or a loop is closer to the rail transit station than linear lines under the same line length. When the line is short, this kind of feeder lines mainly serves the residents near the rail station and is in serious competition with cycling and walking, so the passenger demand for feeder lines is not enough and the accessibility is lower. With the increase of line length, the difference in total passengers served by different shaped lines is narrowing and the advantage of short average travel time of such shaped feeder lines becomes obvious, which leads to higher accessibility. Therefore, the location of the stop at the branch point in Y-shaped and spoon-shaped lines also affects the accessibility.

The performance difference of fixed-route feeder lines caused by shape differences has been systematically considered and studied in this paper, and some ideas are given for thought for transit agencies and managers to design feeder transit systems from the perspective of line shape and routing plans. The results in this paper are obtained under the assumption of certain parameters, but the underlying laws revealed are universal and can be applied to practical problems. There are some deficiencies in this study. Only two-direction routing plans are considered for the three nonlinear lines discussed in this paper. In reality, there are some loop lines and spoonshaped lines that only run clockwise or anticlockwise, and their accessibility could be lower than linear lines under the calculation method in this paper. Also, the values of $\beta$ and the parameters in the demand function are assumed based on a possible situation, so the charts and conclusions in this paper cannot be directly used for transportation planning. Moreover, only the accessibility of the fixed-route feeder lines is studied, but not that of the demand-response feeder lines. Future research can make improvements in these aspects.

\section{Data Availability}

No real data was used to support this study.

\section{Conflicts of Interest}

The authors declare that they have no conflicts of interest.

\section{Acknowledgments}

This research was funded by the National Natural Science Foundation of China (71971021).

\section{References}

[1] S. Chandra, M. E. Bari, P. C. Devarasetty, and S. Vadali, "Accessibility evaluations of feeder transit services," Transportation Research Part A: Policy and Practice, vol. 52, pp. 47-63, 2013.

[2] S. C. Ho, W. Y. Szeto, Y.-H. Kuo, J. M. Y. Leung, M. Petering, and T. W. H. Tou, "A survey of dial-a-ride problems: literature review and recent developments," Transportation Research Part B: Methodological, vol. 111, pp. 395-421, 2018. 
[3] F. Qiu, W. Li, and J. Zhang, "A dynamic station strategy to improve the performance of flex-route transit services," Transportation Research Part C: Emerging Technologies, vol. 48, pp. 229-240, 2014.

[4] F. Errico, T. G. Crainic, F. Malucelli, and M. Nonato, "A survey on planning semi-flexible transit systems: methodological issues and a unifying framework," Transportation Research Part C: Emerging Technologies, vol. 36, pp. 324-338, 2013.

[5] S. N. Kuan, H. L. Ong, and K. M. Ng, "Solving the feeder bus network design problem by genetic algorithms and ant colony optimization," Advances in Engineering Software, vol. 37, no. 6, pp. 351-359, 2006.

[6] L. Quadrifoglio and X. Li, "A methodology to derive the critical demand density for designing and operating feeder transit services," Transportation Research Part B: Methodological, vol. 43, no. 10, pp. 922-935, 2009.

[7] J. A. Pooler, "The use of spatial separation in the measurement of transportation accessibility," Transportation Research Part A: Policy and Practice, vol. 29, no. 6, pp. 421-427, 1995.

[8] P. R. Carleton and J. D. Porter, "A comparative analysis of the challenges in measuring transit equity: definitions, interpretations, and limitations," Journal of Transport Geography, vol. 72, pp. 64-75, 2018.

[9] W. G. Hansen, "How accessibility shapes land use," Journal of the American Institute of Planners, vol. 25, no. 2, pp. 73-76, 1959.

[10] A. Páez, D. M. Scott, and C. Morency, "Measuring accessibility: positive and normative implementations of various accessibility indicators," Journal of Transport Geography, vol. 25, pp. 141-153, 2012.

[11] W. Xu, Y. Ding, J. Zhou, and Y. Li, "Transit accessibility measures incorporating the temporal dimension," Cities, vol. 46, pp. 55-66, 2015.

[12] A. T. Murray, "Strategic analysis of public transport coverage," Socio-Economic Planning Sciences, vol. 35, no. 3, pp. 175-188, 2001.

[13] L. Yang, J. Zhou, O. F. Shyr, and D. Huo, "Does bus accessibility affect property prices?” Cities, vol. 84, pp. 56-65, 2019.

[14] A. Lessa, C. Lobo, and L. Cardoso, "Accessibility and urban mobility by bus in belo horizonte/minas gerais-Brazil," Journal of Transport Geography, vol. 77, pp. 1-10, 2019.

[15] A. Aklilu and T. Necha, "Analysis of the spatial accessibility of addis ababa's light rail transit: the case of east-west corridor," Urban Rail Transit, vol. 4, no. 1, pp. 35-48, 2018.

[16] A. Curl, J. D. Nelson, and J. Anable, "Same question, different answer: a comparison of GIS-based journey time accessibility with self-reported measures from the National Travel Survey in England," Computers, Environment and Urban Systems, vol. 49, pp. 86-97, 2015.

[17] W. Xu and L. Yang, "Evaluating the urban land use plan with transit accessibility," Sustainable Cities and Society, vol. 45, pp. 474-485, 2019.

[18] C. Curtis, E. Ellder, and J. Scheurer, "Public transport accessibility tools matter: a case study of Gothenburg, Sweden," Case Studies on Transport Policy, vol. 7, no. 1, pp. 96-107, 2019.

[19] B. Cui, G. Boisjoly, A. El-Geneidy, and D. Levinson, "Accessibility and the journey to work through the lens of equity," Journal of Transport Geography, vol. 74, pp. 269-277, 2019.

[20] B. Tahmasbi and H. Haghshenas, "Public transport accessibility measure based on weighted door to door travel time," Computers, Environment and Urban Systems, vol. 76, pp. 163-177, 2019.
[21] S. C. M. Geertman and J. R. Ritsema Van Eck, "GIS and models of accessibility potential: an application in planning," International Journal of Geographical Information Systems, vol. 9, no. 1, pp. 67-80, 1995.

[22] Y. J. Wang, Y. Chen, and Q. Li, "Analyzing the change of commuting accessibility by car and its driving forces in Beijing," Jiaotong Yunshu Xitong Gongcheng Yu Xinxi/Journal of Transportation Systems Engineering and Information Technology, vol. 14, no. 5, 2014.

[23] X. Zhu and S. Liu, "Analysis of the impact of the MRT system on accessibility in Singapore using an integrated GIS tool," Journal of Transport Geography, vol. 12, no. 2, pp. 89-101, 2004.

[24] L. A. Merlin, J. Levine, and J. Grengs, "Accessibility analysis for transportation projects and plans," Transport Policy, vol. 69, pp. 35-48, 2018.

[25] M. V. Corazza and N. Favaretto, "A methodology to evaluate accessibility to bus stops as a contribution to improve sustainability in urban mobility," Sustain.vol. 11, no. 3, 2019.

[26] S. Lakhotia, S. Lassarre, K. R. Rao, and G. Tiwari, "Pedestrian accessibility and safety around bus stops in Delhi," IATSS Research, vol. 44, no. 1, pp. 55-66, 2020.

[27] J. Chen, J. Ni, C. Xi, S. Li, and J. Wang, "Determining intraurban spatial accessibility disparities in multimodal public transport networks," Journal of Transport Geography, vol. 65, pp. 123-133, 2017.

[28] J. Jiao, J. Wang, F. Jin, and M. Dunford, "Impacts on accessibility of China's present and future HSR network," Journal of Transport Geography, vol. 40, pp. 123-132, 2014.

[29] L. Quadrifoglio, R. W. Hall, and M. M. Dessouky, "Performance and design of mobility allowance shuttle transit services: bounds on the maximum longitudinal velocity," Transportation Science, vol. 40, no. 3, pp. 351-363, 2006.

[30] H. H. Hochmair, "Assessment of bicycle service areas around transit stations," International Journal of Sustainable Transportation, vol. 9, no. 1, pp. 15-29, 2015.

[31] S. J. Zhang, S. P. Jia, Y. Q. Wang, J. Li, and T. Zhang, "Passenger flow assignment model considering the attraction areas of multiple access modes for urban rail transit stations," Jiaotong Yunshu Xitong Gongcheng Yu Xinxi/Journal of Transportation Systems Engineering and Information Technology, vol. 18, no. 5, pp. 38-45, 2018.

[32] M. Wardman, "Public transport values of time," Transport Policy, vol. 11, no. 4, pp. 363-377, 2004.

[33] S. Tabassum, S. Tanaka, F. Nakamura, and A. Ryo, "Feeder network design for mass transit system in developing countries (case study of Lahore, Pakistan)," Transportation Research Procedia, vol. 25, pp. 3129-3146, 2017.

[34] K. Lättman, L. E. Olsson, and M. Friman, "A new approach to accessibility-examining perceived accessibility in contrast to objectively measured accessibility in daily travel," Research in Transportation Economics, vol. 69, pp. 501-511, 2018.

[35] B. van Wee, "Accessible accessibility research challenges," Journal of Transport Geography, vol. 51, pp. 9-16, 2016.

[36] K. B. Campbell, J. A. Rising, J. M. Klopp, and J. M. Mbilo, "Accessibility across transport modes and residential developments in Nairobi," Journal of Transport Geography, vol. 74, pp. 77-90, 2019. 